\title{
Ingrid Kurz: Simultandolmetschen als Gegenstand der inter- disziplinären Forschung. Wien: WUV-Universitätsverlag, 1996
}

Die relativ wenigen, auf empirischen Untersuchungen basierenden Arbeiten über das Simultandolmetschen waren bislang vor allem in Kurzform in Zeitschriftenartikeln und vereinzelten Beiträgen zu Sammelbänden zugänglich, oder sie lagerten unveröffentlicht in den Bibliotheken von Universitätsinstituten. Deshalb ist es erfreulich, daß nun mit der Druckfassung der Habilitationsschrift von Kurz eine Publikation vorliegt, die nicht nur einen Teilaspekt des komplexen Gegenstandes behandelt, sondern einen nach Schwerpunktbereichen gebündelten interdisziplinären Überblick über das Simultandolmetschen als Forschungsgegenstand bietet. Die Arbeit von Kurz ist im übrigen nach der Veröffentlichung der Dissertation von Pöchhacker (1994) bereits die zweite veröffentlichte Monographie über das Simultandolmetschen, die der Werkstatt des Wiener Instituts für Übersetzer- und Dolmetscherausbildung zu verdanken ist.

Die Arbeit enthält sechs Hauptkapitel, ergänzt durch Einleitung, Zusammenfassung und Bibliographie. In den Hauptkapiteln werden die von anderen Disziplinen aufgestellten Theorien und Hypothesen kurz dargestellt und dann auf ihre Gültigkeit für das Simultandolmetschen geprüft. Die Überprüfung erfolgt zumeist anhand von Überblicken über empirische Untersuchungen, wobei die Auswahl der referierten Literatur zum Teil von "persönlichen Vorlieben" (S. 15-16) für Untersuchungen geprägt ist, die Kurz für die weitere Erforschung des Simultandolmetschens als wegweisend betrachtet, nämlich vor allem psychologische und neurophysiologische Ansätze. Es handelt sich bei der vorliegenden Arbeit um ein persönliches Buch, insofern als sich die Verfasserin hierbei in hohem Grade auf eigene, im Laufe von mehr als zwanzig Jahren Beschäftigung mit dem Simultandolmetschen entstandene Untersuchungen stützt. U.a. dadurch unterscheidet sich das Buch von einer kürzlich erschienenen Sammlung, die ebenfalls empirische Untersuchungen über das Simultandolmetschen vorstellt (Lambert/Moser-Mercer 1994).

Kapitel 1 (Zur Geschichte des Simultandolmetschens) skizziert den Werdegang der neuen Technik. Seit dem ersten Einsatz einer Simultananlage bei der International Labor Conference 1927 - die Nürnberger Kriegsverbrecherprozesse waren nicht, wie oft behauptet wird, die erste Gelegenheit, wohl aber die spektakulärste - setzte sich das Simultandolmetschen im Laufe von nur etwa 25 Jahren durch, obwohl das simultane Dolmetschen zunächst von maßgeblichen Persönlichkeiten als unzuverlässig und als qualitativer Rückschritt im Vergleich zum Konsekutivdolmetschen betrachtet wurde. Kurz stellt fest, daß Einrichtungen wie die UNO oder die EU ohne die Entwicklung der Simultan- 
technik gar nicht hätten entstehen können (S.34) und daß heute das Simultandolmetschen bei internationalen Konferenzen etc. "nicht mehr wegzudenken" sei (S. 19) - eine vielleicht etwas zu optimistische Beurteilung angesichts der steigenden Tendenz, Englisch als internationale Arbeitssprache zu benutzen und auf Dolmetscherdienste zu verzichten.

Kapitel 2 (Zur Qualität der Dolmetschleistung - Beiträge der Translationstheorie und Textlinguistik zum Simultandolmetschen und ihre empirische Überprüfung) befaßt sich zunächst mit der Frage, wieweit die Aussagen der allgemeinen Translationstheorie und inbesondere der Skopostheorie von Reiß/ Vermeer (1984), die Anspruch auf Gültigkeit für das Übersetzen und das Dolmetschen erheben, tatsächlich für das Simultandolmetschen relevant sind. Zwar erkennt Kurz allgemeine Aussagen der Theorie über die Wichtigkeit von Skopos, Situation und Kohärenz als beinahe triviale Selbstverständlichkeit an, aber insgesamt betrachtet sie die Relevanz der Theorie für das Simultandolmetschen als sehr begrenzt. Diese Einschätzung scheint mir insofern nicht ganz gerechtfertigt zu sein, als gerade der Prozeß der kritischen Abgrenzung gegenüber der Skopostheorie zu grundlegenden Erkenntnissen wie z.B. der stärkeren Ausgangstext- und Situationsgebundenheit von Simultandolmetschungen geführt hat.

Einen starken Einfluß auf das Simultandolmetschen hat die Textlinguistik ausgeübt. De Beaugrande/Dresslers (1981) bekannte Textualitätskriterien und die Schlüsselrolle, die die Autoren dem "Textbenützer" zuweisen, haben die Erkenntnis befördert, daß sich die Frage nach der Qualität von Zieltexten beim Dolmetschen nur durch Rezipientenbefragungen erhellen läßt. Und hier zeigen empirische Untersuchungen, daß es “den” Rezipienten nicht gibt und daß Qualitätsparameter wie Kohärenz und Kohäsion von verschiedenen Rezipientengruppen ganz unterschiedlich bewertet werden.

So ließ Kurz mittels Fragebogen drei verschiedene Rezipientengruppen (Mediziner, Techniker und Teilnehmer an einer Europaratskonferenz) die Wichtigkeit einer Reihe von Qualitätskriterien beurteilen, die Bühler (1986) bei einer Befragung von AIIC-Dolmetschern benutzt hatte. Ein nicht verwunderliches Ergebnis war, daß die von Bühler befragten Dolmetscher generell höhere Qualitätsanforderungen stellten als die drei Gruppen von Konferenzteilnehmern. Interessant ist dagegen, daß die festgestellten Unterschiede in der Beurteilung der Qualitätsparameter durch die letzteren Gruppen entscheidend von den Formen der Informationsvermittlung abhängen, die im Rahmen unterschiedlicher Konferenztypen dominieren. Beispielsweise erklärt Kurz die relativ niedrige Bewertung des logischen Zusammenhangs ("Kohärenz") durch die Techniker damit, daß technische Vorträge häufig durch visuelles Begleitmaterial ergänzt werden. Und die noch niedrigere Bewertung von Kohärenz 
durch die Teilnehmer an der Europaratskonferenz wird dadurch plausibel, daß den Teilnehmern Dokumente und Arbeitsunterlagen im voraus zur Verfügung stehen, während auf der Konferenz selbst Informationsaustausch und Diskussion dominieren, also spontane Beiträge, an die weniger strenge Kohärenzanforderungen gestellt werden. Eine Systematisierung von Zusammenhängen dieser Art ist übrigens von Pöchhacker (1994) vorgeschlagen worden, und da Kurz an anderer Stelle im Buch auf diese Publikation verweist, ist es verwunderlich, daß Pöchhackers "Textprofile" in der Übersicht über verschiedene "Text-, Redner- und Rezipiententypologien" (Kapitel 2.6.) nicht erwähnt werden.

Unter den in Kapitel 3 (Für das Simultandolmetschen relevante Ergebnisse der kognitionspsychologischen Forschung) dargestellten kognitionspsychologischen Erkenntnissen ist besonders hervorhebenswert, daß komplexe Fertigkeiten wie das Simultandolmetschen in Teilkomponenten zerlegbar sind, die unabhängig voneinander geübt werden können; daß bei der Mustererkennung die Top-down-Verarbeitung, die Kontext und Allgemeinwissen einbezieht, von den Experten zunehmend als vorrangig betrachtet wird; daß sich die begrenzte Speicherkapazität des aktivierten Gedächtnisses mit der Bedeutungshaltigkeit des Materials ändert (Ausweitung durch "Chunking" bei Experten im Vergleich zu Anfängern); daß durch Übung Aufmerksamkeitsgrenzen erweitert werden können.

Als Beispiele für Bereiche, die sich für eine unmittelbare Anwendung dieser Erkenntnisse auf das Simultandolmetschen anbieten, nennt Kurz folgende: 1. Die Verarbeitung ein- und zweisprachiger Kommunikation (die nach Meinung der Experten keine prinzipiellen Unterschiede aufweist), 2. Die Bedeutung des Kontextes für das Mustererkennen (hier ist u.a. Antizipationsfähigkeit wichtig), 3. Aufmerksamkeitsverteilung - Giles "effort model", 4. Übung und Automatisierung von dolmetschrelevanten Fertigkeiten, 5. Konferenzterminologie und -phraseologie (die durch gezielte Übung automatisiert werden kann). Den unter 4. genannten Bereich exemplifiziert Kurz ausführlich im nächsten Kapitel.

Kapitel 4 (Empirische Untersuchungen zum Einfluß der Übung auf dolmetschrelevante Parameter) beleuchtet die Übung von Teilfertigkeiten. Zur Erlernung der Teilfertigkeit des simultanen Sprechens und Hörens in der Dolmetscherausbildung sind verschiedene Übungen vorgeschlagen worden. Kurz argumentiert überzeugend für die Ansicht, daß diese Übungen überwiegend nicht sinnvoll und teilweise sogar kontraproduktiv sind, weil sie meist mit dem eigentlichen Ausbildungsziel nichts zu tun haben. Abzulehnen sind daher Phonemic Shadowing, Dual-task-training (bei nicht dolmetschrelevanten Aufga- 
bentypen), Digit processing, Lag exercises (mit einem vorgegebenen fixen zeitlichen Abstand).

In ihrer Dissertation (Pinter 1969), einer empirischen Untersuchung des Übungseinflusses auf die Fertigkeit des simultanen Sprechens und Hörens, verwendete die Verfasserin sinnvolles einsprachiges Material, wenn auch aus methodischen Gründen nicht in der Form fortlaufenden Kontextes. Hier konnte der Übungseinfluß durch den Vergleich der Leistungen von vier verschiedenen Versuchsgruppen mit sehr unterschiedlichem Übungsgrad empirisch nachgewiesen werden. 1990 testete Kurz den Übungseinfluß erneut, nun jedoch in Form einer Längsschnittuntersuchung mit fünf Probanden (Dolmetschanfängern), deren Leistungen vor und nach einer fünfmonatigen Übungszeit verglichen wurden. In dieser Untersuchung wurde dasselbe Versuchsmaterial wie in der Referenzstudie von 1969 benutzt, das aus Aufgabentypen von steigendem Schwierigkeitsgrad bestand. Der Ergebnisvergleich dokumentierte wiederum eine signifikante Leistungssteigerung durch Übung, und zwar am deutlichsten bei den kognitiv anspruchsvolleren Aufgabentypen. Die Leistungsverbesserung bei diesen führt Kurz auf die weitgehende Automatisierung des gleichzeitigen Sprechens und Hörens (und zweckmäßige Aufmerksamkeitsverteilung) nach einem Semester Simultandolmetschen zurück.

Eine weitere dolmetschrelevante Teilfertigkeit ist die Assoziationsflüssigkeit, die mit zunehmender Sprachbeherrschung steigt. Kurz testete die Assoziationsflüssigkeit von Übersetzer- und Dolmetscherstudierenden in verschiedenen Ausbildungsstadien sowohl in der Muttersprache als auch in der ersten Fremdsprache der Versuchspersonen, um den Einfluß der Übung auf die Sprachbeherrschung festzustellen. Auf der Grundlage der Anzahl von Reaktionen auf die Reizwörter sprechen bzw. to speak, die innerhalb von zwei Minuten aufzuschreiben waren, wurde für alle Probanden ein sogen. "Balance Score" errechnet, aus dem hervorging, daß bei allen Versuchsgruppen die Assoziationsflüssigkeit in der Muttersprache signifikant besser war als in der Fremdsprache. Es gab m.a.W. unter den Probanden keine "balanced bilinguals", die zwei Sprachen gleich gut beherrschen. Allerdings war der Leistungsunterschied zwischen beiden Sprachen bei den fortgeschrittensten Dolmetscherstudierenden als Folge der Übung wesentlich geringer als bei einer Kontrollgruppe von Psychologiestudierenden und kam dementsprechend in einem niedrigeren Balance Score zum Ausdruck. Da die Sprachbeherrschung und die linguistische Dominanz mit dieser Methode objektiv meßbar ist, empfiehlt Kurz sie zur Erprobung als Teil von Eignungsprüfungen an Dolmetscherinstituten.

In Kapitel 5 (Persönlichkeitsuntersuchungen an Dolmetschern) gibt Kurz zunächst einen Überblick über bereits existierende Persönlichkeitsuntersuchungen an Dolmetschern, stellt anschließend einige von der Psychologie 
entwickelte Testmethoden vor und beschreibt deren Anwendung in einer eigenen Untersuchung über Angst bei Konferenzdolmetschern.

Um Aufschluß über Streßbewältigung beim Simultandolmetschen zu gewinnen, testete Kurz das Persönlichkeitsmerkmal Angst bei 32 Konferenzdolmetschern mit Hilfe des "State-Trait Anxiety Inventory" (STAI), das mit Hilfe zweier Selbstbeschreibungsskalen die allgemeine Ängstlichkeit (TraitAngst) und die als Reaktion auf eine bedrohliche Situation empfundene Zustandsangst (State-Angst) mißt. Bei der State-Angst-Skala mußten die Versuchspersonen angeben, wie sie sich unmittelbar vor dem Dolmetschen fühlen. Die Antworten der Konferenzdolmetscher wurden mit den Stichprobenwerten für die jeweiligen Referenzgruppen (Frauen bzw. Männer in der Altersgruppe 30-59 Jahre) verglichen. Die Ergebnisse wurden von Kurz im Rahmen der "Ergopsychometrie", einer von der Sportpsychologie entwickelten Methode zur Leistungsdiagnostik, folgendermaßen interpretiert: Die Mehrheit der Konferenzdolmetscher, deren generelle Ängstlichkeit signifikant geringer war als die der Referenzgruppe, wird voraussichtlich auf Belastung mit Leistungsanstieg reagieren. Eine kleinere Gruppe von Dolmetschern hatte höhere Werte für Trait-Angst als für Zustandsangst, da aber die Zustandsangst deutlich niedriger war als bei der Referenzgruppe, vergleicht Kurz diese Dolmetscher mit besonders leistungsstarken Sportlern, die ihre Angst besser unter Kontrolle haben und in Streßsituationen ihre Leistung erhöhen. Die befragten Dolmetscher gehören also nicht zum Typ des "Trainingsweltmeisters", der unter Belastung in der realen Situation abbaut.

Die in Kapitel 6 (Neurophysiologische Forschungsergebnisse) referierten Forschungsergebnisse betreffen die Schwerpunkte Lateralität sowie die elektrische Aktivität des Gehirns bei komplexen Denkvorgängen.

Die Aphasieforschung hat viele Indizien für eine linkshemisphärische Sprachrepräsentation bei rechtshändigen Erwachsenen gefunden, während bei Linkshändern eine bilaterale Sprachrepräsentation anzunehmen ist. Die zerebrale Organisation der Sprache bei Zwei- und Mehrsprachigen ist jedoch nach Erkenntnissen der Neurolinguistik und Psycholinguistik anders, und zwar symmetrischer als bei Einsprachigen. Fingerklopfversuche deuten auf eine stärkere Beteiligung der rechten Hemisphäre hin, besonders bei Personen, die die Zweitsprache erst nach der frühen Kindheit erlernt hatten. Durch Versuche mit dichotischem Hören und Fingerklopfen wurde auch an der Dolmetscherschule in Triest die Annahme einer symmetrischen Sprachrepräsentation bei fortgeschrittenen Dometschstudentinnen erhärtet.

Die letzte, im vorliegenden Buch referierte Untersuchung ist ein Selbstversuch, bei dem die Gehirnaktivität der Verfasserin aufgezeichnet wurde, während sie u.a. "mentales", d.h. stummes Simultandolmetschen ausführte. 
Mit der Methode des "EEG-Probability-Mapping" werden mit Computerhilfe "Kohärenzen", d.h. potentielle funktionale Verknüpfungen zwischen zwei Hirnregionen berechnet und auf dem Bildschirm graphisch dargestellt. In dem Selbstversuch wurden auf diese Weise charakteristische EEG-Veränderungen beim Dolmetschen in die Muttersprache und in die Fremdsprache, Musikhören und Kopfrechnen im Vergleich zum Ruhe-EEG sichtbar gemacht. Die Ergebnisse zeigen vor allem, daß (bei Rechtshändigkeit) die rechte Hemisphäre durch das Simultandolmetschen in die Fremdsprache mehr beansprucht wird als durch das Dolmetschen in die Muttersprache. Damit bestätigen sich Ergebnisse von Forschern, die mit völlig anderen Methoden gearbeitet haben.

Das vorliegende Buch ist vor allem für zwei Zielgruppen empfehlenswert. Zunächst ist es allen zu empfehlen, die sich einen lesbaren Überblick über wesentliche Bereiche und aktuelle Strömungen innerhalb der Forschung zum Simultandolmetschen wünschen. Dies könnten z.B. praktizierende Dolmetscher und in der Lehre Tätige sein oder auch Vertreter anderer Disziplinen, die an einer Orientierung interessiert sind. Das Buch ist kein Bericht aus dem Elfenbeinturm, sondern aus einer praxisbezogenen Perspektive in verständlicher Sprache geschrieben. Besonders aber ist die Arbeit für den potentiellen wissenschaftlichen Nachwuchs an den universitären Übersetzer- und Dolmetscherinstituten geeignet. Angehende Diplomanden und Doktoranden werden in diesem Buch eine Fülle von Anregungen für Projektthemen und -methoden finden. Im Hinblick auf die letztere Zielgruppe hätte man sich im übrigen gewünscht, daß zitierte Literatur und Literatur, auf die mehrfach verwiesen wird, in der Bibliographie zu finden ist, auch wenn es sich um eine Auswahlbibliographie handelt.

Insgesamt ist zu sagen, daß das Buch durch die Konzentration auf empirische Untersuchungen dazu beiträgt, eine große Lücke zu schließen. Es stellt intersubjektiv nachprüfbare Daten bereit, die die noch junge Dolmetschwissenschaft zu ihrer Weiterentwicklung dringend braucht. Und die verständliche Darstellung wird das Ihre tun, um die Berührungsängste von Sprachwissenschaftlern gegenüber neuen und neusten Methoden der interdisziplinären Forschung zu mindern. Es ist ein nützliches und zugleich ein inspirierendes Buch.

\section{Literatur:}

Lambert, Sylvie \& Moser-Mercer, Barbara (eds.) (1994): Bridging the gap: Empirical research in simultaneous interpretation. Amsterdam/Philadelphia: Benjamins.

Pöchhacker, Franz (1994): Simultandolmetschen als komplexes Handeln. Tübingen: Narr. 
